\title{
Le Théâtre Anglois d'Antoine de La Place (1746-1749), ou la difficile émergence du théâtre de Shakespeare en France
}

\section{Christian Biet}

\section{(2) OpenEdition \\ 1 Journals}

\section{Édition électronique}

URL : http://journals.openedition.org/shakespeare/533

DOI : 10.4000/shakespeare.533

ISSN : 2271-6424

Éditeur

Société Française Shakespeare

\section{Édition imprimée}

Date de publication : 1 novembre 2000

Pagination : 27-46

ISBN : 2-84269-407-4

\section{Référence électronique}

Christian Biet, «Le Théâtre Anglois d'Antoine de La Place (1746-1749), ou la difficile émergence du théâtre de Shakespeare en France ", Actes des congrès de la Société française Shakespeare [En ligne], 18 | 2000, mis en ligne le 01 novembre 2007, consulté le 22 avril 2019. URL : http:// journals.openedition.org/shakespeare/533; DOI : 10.4000/shakespeare.533 


\section{S H A K E S P E A R E \\ \& L A F R A N C E}

Société Française Shakespeare

Actes du Congrès de 2000

* * *

Textes réunis et présentés par

Patricia DORVAL

publiés sous la direction de Jean-Marie MAGUIN 
Colloque honoré d'une subvention du Ministère de l'Éducation Nationale, de la Recherche et de la Technologie

Site Internet $:<$ http://alor.univ-montp3.fr/SFS/> Liste de diffusion : <sfs-1@smr1.univ-montp3.fr>

Tous droits de traduction, de reproduction et d'adaptation réservés pour tous les pays.

(C) 2000. Société Française Shakespeare, École Normale Supérieure, 45 rue d'Ulm, 75005 Paris.

ISBN 2-84269-407-4 
LE THÉÂTRE ANGLOIS D'A NTOINE DE LA PLACE (1746-1749) 1, OU LA

D I F F I C I L E É M E R G E NCE DU THÉ ÂTRE DE S HA KES PEARE EN FRANCE

Il importe peu que [Shakespeare] ait travaillé dans un goût différent du nôtre : cette raison même ne doit servir qu'à redoubler notre curiosité. ${ }^{2}$

Si le XVIIe siècle français fut, dans son ensemble, absolument ignorant des pièces élisabéthaines, le XVIIIe siècle, peu à peu, grâce à Voltaire et à Saint-Evremond, superbes exilés, se pencha sur le théâtre anglais. Aux références antiques, italiennes et espagnoles, on dut bien ajouter, non sans réticences ni résistance, celles d'un monde radicalement exogène, fait de spectacle, de sang et de diversité. Et il fallut bien un siècle pour que Shakespeare s'installât sur la scène française, largement bridé par une traduction craintive.

\section{Le Théâtre anglois, un théâtre pour les lecteurs}

Pierre-Antoine de La Place est le premier traducteur français à avoir publié une traduction / adaptation du thêatre de Shakespeare et en particulier d'Othello, en 1746-1749, premier texte traduit dans son recueil de huit volumes, dont $\mathrm{j}$ 'ai déjà parlé ailleurs ${ }^{3}$. Et ce que l'on constate bien vite, c'est que la tragédie, enjeu massif du 
recueil, est bien différente selon qu'on se place d'un côté et de l'autre du Channel et du XVIIe siècle.

La Place se donne pour mission de rendre compte de la tragédie anglaise pour son public propre, un public de lecteurs et non encore de spectateurs (il insiste sur le fait que ce texte traduit ne peut être représenté), un public qui lit la tragédie au regard des auteurs antiques et modernes: Aristote est convoqué au même titre que Shakespeare, Corneille et Voltaire. Ainsi, transcrire la passion dans le théâtre tragique, c'est non seulement lire d'une certaine manière la tradition élisabéthaine, mais aussi tenir compte du théâtre classique du XVIIe siècle fondé sur la Poétique, et du théâtre contemporain situé dans un entre-deux, considérant, à la manière de Gresset et de Crébillon père, que le «sanglant» peut être un ressort de la tragédie. Traduire le théâtre anglais, c'est donc transcrire, informer, et nécessairement adapter, sous peine de n'être pas compris du lecteur, c'est faire fonctionner le texte élisabethain et surtout shakespearien dans les normes préexistantes du genre tragique français, sous peine de choquer ou de n'être point compris.

Comment faire état du goût anglais ? Comment, en dix pièces véritablement traduites, juger Shakespeare ? Comment le traduire, malgré tout? Faut-il résumer ce qui n'est pas traduisible (l'ensemble du théâtre shakespearien est résumé dans des analyses) ? Comment représenter le paysage dramatique anglais en traduisant treize autres pièces, de Ben Johnson à Steele ou Addison, en passant par Otway? Comment expliquer la fondamentale étrangeté du théâtre anglais au moment où règne en France une évidente anglomanie ? Pour La Place, la poésie de Pope - dont il cite, dans son long «Discours» et sa Vie de Shakespeare, le jugement sur Shakespeare -, les essais de Locke, les romans de Defoe sont maintenant connus et appréciés. Il serait donc paradoxal, compte tenu de la hiérarchie des genres, que la tragédie ne le soit point. Et puisque le goût des Français lettrés pour l'anglais change peu à peu, il est maintenant nécessaire de faire le point sur la culture anglaise dans son ensemble, ne serait-ce que pour l'histoire, ne serait-ce aussi que pour prendre date. La Place, dans son «Discours sur le théâtre anglais» de 1746, qu'il inscrit en tête de ses huit volumes de traductions, se justifie donc de son choix nécessaire et de sa pratique de traducteur, et c'est cette justification qu'on analysera ici. 


\section{Présence de l'Angleterre et modification du goût}

Je voudrais, auparavant, faire un détour par Voltaire qui, comme d'habitude, place agréablement et clairement les enjeux. Pour Voltaire, Shakespeare et le théâtre d'Outre-Manche sont loin d'être négligeables, mais pour autant, ils sont «barbares».

C'est Shakespeare qui, tout barbare qu'il était, mit dans l'anglais cette force et cette énergie qu'on n'a jamais pu augmenter depuis sans l'outrer, et par conséquent sans l'affaiblir. D'où vient ce grand effet de la poésie, de former et fixer enfin le génie des peuples et de leurs langues? La cause en est bien sensible : les premiers bons vers, ceux même qui n'en ont que l'apparence, s'impriment dans' la mémoire à l'aide de l'harmonie. Leurs tours naturels et hardis deviennent familiers; les hommes, qui sont tous nés imitateurs, prennent insensiblement la manière de s'exprimer, et même de penser, des premiers dont l'imagination a subjugué celle des autres. ${ }^{4}$

Ainsi, les barbares peuvent faire de bons vers à condition qu'ils aient l'énergie, la force, le génie en un mot, et qu'ils soient harmonieux. C'est d'ailleurs la raison pour laquelle Voltaire luimême, dans la dix-huitième Lettre philosophique, «sur la tragédie», de 1734, se mêle de traduire le monologue d'Hamlet, avec d'ailleurs un certain talent. Durant son séjour en Angleterre vers 1727-1728, Voltaire avait donc décourvert le théâtre anglais et compris que Shakespeare avait, comme plus tard Corneille en France, «créé le théâtre», là-bas. "Il avait un génie plein de force et de fécondité, de naturel et de sublime, sans la moindre étincelle de bon goût, et sans la moindre connaissance des règles». Le travail du bon lecteurspectateur français consistera donc à privilégier le bon grain et à rejeter l'ivraie, en d'autres termes à considérer ce qui est naturel, sublime, fort et fécond, et à ignorer ou condamner ce qui choque la bienséance et la vraisemblance, et ce qui s'oppose trop aux règles, qui elles-mêmes découlent du vraisemblable. Les «belles scènes» et «les morceaux si grands et si terribles» devront donc être privilégiés, choisis par le lecteur-spectateur, au détriment du bas, du grotesque, de la farce et du «monstrueux» propres au genre théâtral anglais. Et le grand tort des auteurs anglais du règne de Charles II, c'est de ne 
pas avoir su trier, sans compter qu'ils n'avaient ni la force, ni l'énergie de leur modèle. Ainsi, poursuit Voltaire, il faut savoir écarter les erreurs anciennes (celles de Shakespeare) et modernes (celles de ses imitateurs, encore plus scandaleuses) et savoir traduire les «endroits frappants».

Vous vous plaindrez sans doute que ceux qui jusqu'à présent vous ont parlé du théâtre anglais, et surtout de ce fameux Shakespeare, ne vous aient encore fait voir aucun de ces endroits frappants qui demandent grâce pour toutes ses fautes. 5

S'il est possible, donc, d'entendre Shakespeare, ce ne peut être qu'en morceaux, et sans le traduire mot pour mot: «malheur aux faiseurs de traductions littérales qui, en traduisant chaque parole, énervent le sens. C'est bien là qu'on peut dire que la lettre tue et que l'esprit vivifie». En soumettant ainsi, traduits en alexandrins, un passage d'Hamlet et un morceau de Dryden, Voltaire choisit. Et ce choix se justifie par la forme et le génie anglais : en effet, Voltaire affirme que les poètes anglais, eux-mêmes, sont orfèvres en pièces disjointes. «C'est dans ces morceaux détachés que les tragiques anglais ont jusqu'ici excellé : leurs pièces presque toutes barbares, dépourvues de bienséance, d'ordre, de vraisemblance, ont des lueurs étonnantes au milieu de cette nuit». Il est dans le naturel du théâtre anglais d'être composite, et dans le naturel du spectateur français de choisir ce qui correspondra à ses propres canons. Pour autant, parce que le naturel anglais est ainsi fait, les dramaturges modernes d'Outre-Manche ont tort de vouloir imiter la régularité française et deviennent froids à mesure qu'ils forcent leur nature : «il faut avouer que les échasses du style figuré, sur lesquelles la langue anglaise est guindée, élèvent aussi l'esprit bien haut, quoique par une marche irrégulière». À chacun sa langue, à chacun son génie, et seuls certains passages dans lesquels une grandeur et une harmonie universelles sont patentes, peuvent traverser la Manche, dans un sens ou dans l'autre.

Voilà donc d'où part La Place : d'une habitude du choix, légitimée par un jugement sur le génie universel des peuples, luimême fondé sur l'idée de génie individuel, d'énergie et de force. Mais comment faire lorsqu'on a vocation à ne pas (trop) choisir ? Que faire lorsqu'il faut présenter toute une tragédie sans se limiter à un morceau à caractère universel ? Là est l'obstacle principal que 
doit franchir La Place. Pour lui, comme pour Voltaire et comme pour Crébillon, partiellement «traducteur» de La Tempête, le théâtre anglais élisabéthain est fondamentalement étranger, voire étrange. Cependant, il faut bien dire que ce théâtre plaît Outre-Manche, et depuis fort longtemps: en 1746 Shakespeare est toujours joué, apprécié, adulé à Drury Lane. Quelles que soient les réticences françaises, il n'est plus possible, comme à la fin du XVIIe siècle, de négliger ce succès et de renvoyer les Anglais à leur barbarie. D'autres genres, en particulier philosophique et romanesque, ont montré que les auteurs anglais devaient être fort bien considérés et de nombreux auteurs français, depuis le début du XVIIIe siècle, et plus particulièrement depuis la Régence anglomane, ont voulu donner du théâtre anglais une image plus favorable en l'adaptant. On sait que Voltaire, entre autres, s'y est appliqué, avec Zaïre, en 1733, mais aucun n'a traduit Shakespeare de manière exhaustive ou au moins systématique.

La Place assure avoir donc attendu que «nos» grands auteurs (Voltaire ? Saint-Évremond ? pourquoi pas Montesquieu ?) se soient livrés à cet exercice, mais rien n'est venu. Il s'agit donc de combler un vide, de fournir au lecteur français les pièces d'un dossier qu'il pourra comparer avec le corpus culturel contemporain, c'est-à-dire avec les théâtres français, latin et grec qu'il connaît nécessairement, «ne serait-ce qu'à titre de curiosité» ${ }^{6}$. Jusqu'ici, on a en effet traduit ou adapté de nombreux et «bons écrits Anglais [...] que d'habiles traducteurs ont fait passer avec applaudissements dans notre langue depuis quelques années» ${ }^{7}$; mais ces écrits ne révèlent rien de la tragédie ou de la comédie.

\section{Comment juger Shakespeare ?}

En prenant comme autorité le R.P. Brumoy ${ }^{8}$, La Place affirme vouloir faire le tour, en huit volumes, d'une évolution d'un siècle, de Shakespeare à Dryden. Le recours à Brumoy n'est pas innocent et le théâtre anglais serait donc jugé à la manière du théâtre des grecs : en effet, le Théâtre des Grecs, publié en 1730 par Brumoy, célèbre jésuite, avec de nombreuses éditions durant tout le XVIIIe siècle est un succès considérable. Ainsi, le public a besoin de comprendre, au même titre qu'il voulait connaître Eschyle, Euripide, et surtout Sophocle, l'usage que les Anglais, "nos voisins», font d'un art majeur qu'ils tiennent des grecs «ainsi que nous» ${ }^{9}$. À travers la traduction ou l'adaptation des œuvres grecques, latines et anglaises, nous assistons donc à une mise en place de la modernité en matière 
de théâtre : mise en place de la tragédie antique dans la modernité avec la découverte du curieux Eschyle et du terrible Sénèque, et tensions de la modernité avec Shakespeare.

Mais la modernité repose aussi sur une méthode raisonnable qui consiste à exercer un choix, à renoncer à l'archaïsme et à rejeter ce qui ne convient pas au monde tel qu'il est, à ses formes et à son goût. Et en matière de traduction, la modernité exclut ainsi toute littéralité, au nom du goût, de la raison et de la vraisemblance.

$\grave{A}$ bien lire Brumoy, on s'aperçoit donc que le travail d'un traducteur est surtout un travail de présentation, de disposition et de choix. Dans le Théâtre des Grecs de 1730, tout n'est pas traduit, bien au contraire ; l'auteur s'interroge sur l'intérêt de faire connaître telle ou telle scène, en complète infraction avec les règles tragiques communément admises, et décide de résumer les passages en expliquant sa censure, généralement au nom des bienséances et de la vraisemblance, autrement dit du contrat de lecture et de vision du spectacle que chaque spectateur a coutume de passer avec le genre qu'il entend voir : ici, la tragédie régulière.

Lire une traduction du grec, c'est ainsi considérer les auteurs grecs comme des auteurs ayant constitué généralement une ébauche que leur siècle ne pouvait absolument maîtriser. Dès lors, le traducteur peut à la fois signaler les imperfections, les dénoncer, et même les corriger : c'est en se référant à cette jurisprudence esthétique que La Place va travailler.

\section{Curiosité, tolérance et universalité esthétiques, dans l'intérêt de tous}

La nation française, abandonnant son regard méprisant pour ce qui n'est pas étroitement de son goût et conforme à ses mœurs, doit, selon La Place, comprendre que le goût épuré qu'elle tient de ses études sur Aristote et du travail élaboré sur la notion de vraisemblance, ne doit pas tuer l'art au nom d'un excessif raffinement. Il s'agit maintenant de comprendre ce qu'un peuple éclairé, proche du nôtre au moins géographiquement, a pu faire de la tradition antique commune aux deux peuples, quitte à le juger par la suite. On sent là l'influence des Lettres Philosphiques... Partant de l'idée que l'Angleterre est maintenant reconnue comme une nation éclairée et que son peuple tout entier ne peut être dupe d'un mauvais auteur, La Place peut donc affirmer qu'il y a une valeur bien réelle dans les pièces de Shakespeare : 
Eh, comment se persuader qu'un peuple
entier soit la dupe d'un faux mérite ?

Ce mérite peut être exagéré, mais il existe néanmoins :

Il importe peu que [Shakespeare] ait travaillé dans un goût différent du nôtre : cette raison même ne doit servir qu'à redoubler notre curiosité. ${ }^{11}$

C'est au nom de la perfection universelle de l'Art, de son unité, qu'il faut étudier les sommets de l'art des autres nations, même si l'abord en est difficile ou absolument étranger. Et c'est au nom de la curiosité moderne, raisonnable et éclairée, qu'il est utile de lire Shakespeare. Universalité, curiosité pour le génie particulier d'un peuple et d'une nation maintenant sortie de la barbarie, c'est entre ces deux termes que La Place évolue.

Pour les qualités ou les particularités propres à la nation anglaise, le traducteur les explique par le terroir, le climat et le système social, mais tient aussi à remarquer qu'il n'y a finalement d'étranger que le traitement esthétique des passions, mais affirme parallèlement que les passions elles-mêmes résistent à la distinction entre génie français et génie anglais. Le goût d'une nation peut donc former ou déformer l'apparence de son théâtre, les conditions sociales de représentation peuvent parfois favoriser les mauvais penchants de la populace - Shakespeare ne devait-il pas gagner sa vie grâce au public mêlé de l'époque, et donc sacrifier à sa bassesse ? - il n'en est pas moins vrai que «la nature des choses» est exactement représentée dans les chefs-d'œuvre. Autrement dit, sous les diversités de l'esthétique, sous les particularités nationales, le naturel, le sublime et l'harmonie restent universels.

Le vrai et le beau ne sont qu'un, [bien que ce principe général reçoive] des modifications relativement au génie, aux mœurs, aux usages, au goût même des différents pays. ${ }^{2}$

Mais plutôt que de renvoyer chacun chez soi en privilégiant quelques morceaux d'universalité, La Place semble alors imaginer un progrès esthétique capable de permettre une évolution de chacune des deux formes théâtrales. Il a probablement lu, en plus de Voltaire, Dryden et ses Essays on Dramatic Poesy et postule que les 
deux systèmes tragiques peuvent être réformables l'un par l'autre. Puisque le français semble être esclave de l'art, et l'anglais esclave de la Nature et du Spectacle, il est temps, semble-t-il, que l'un et l'autre se rejoignent en renonçant à leur propre esclavage.

Shakespeare, particulièrement représentatif de son temps, a puisé, peut-être avec excès, dans le sein de la nature - Pope, auteur légitime s'il en est, en convient dans sa préface de 1728 aux Euvres de Shakespeare, assure La Place.

Jamais poète n'a commandé aux passions avec plus d'empire, et jamais empire n'a été plus grand. ${ }^{13}$

Le lecteur français devra ainsi comprendre que tout contact avec Shakespeare le mettra immédiatement (sans médiation aucune) au contact de la rudesse des passions et de leur incroyable chaleur sanglante. Il devra, lors de sa lecture, tenir compte du public anglais : savoir que ce public possède un caractère rêveur ${ }^{14}$; qu'il n'est pas composé de Grands et de riches, comme le dira Madame Riccoboni, mais de bourgeois et de peuple; que les horreurs de la scène le tirent de son ennui et de sa rêverie; que la représentation spectaculaire de la passion des rois fait que ce même public néglige le vraisemblable ; et que les faits tels qu'il les connaît par l'histoire et par la tradition sont ceux qu'il veut sur scène, sans préparation, avec brutalité, et variété ${ }^{15}$. C'est un fait, «les coeurs de tous les peuples n'ont pas le même unisson», s'ils ont les mêmes passions.

Cependant, il y a beaucoup à apprendre du cœur des autres... Il faut ainsi retenir «la vérité des sentiments» plus que leur exhibition relative au temps et au public, ce qui pourra, le cas échéant, donner lieu à une meilleure compréhension des choses, à une évolution des mentalités, voire à un progrès dans les arts.

Gardons-nous donc de condamner sans retour aujourd'hui ce que nos neveux applaudiront peut-être un jour. ${ }^{16}$

\section{La traduction et le progrès des arts}

D'ailleurs, les Français ne commencent-ils pas à ensanglanter la scène, avec Gresset (Édouard) et Crébillon père («Corneille avait le Ciel, Racine la Terre, il me reste l'Enfer») ? Ne discutent-ils pas la théorie des unités sous la houlette des Modernes (Houdar de La 
Motte en particulier) ? Et cela ne témoigne-t-il pas d'un progrès possible dans les arts, comme on l'a vu dans les sciences? De nouvelles ressources sont trouvées, de nouvelles routes sont découvertes dans l'exploration des replis du genre humain et il convient d'espérer plutôt que de s'en indigner...

La Place arrive en 1746, à un moment où le progrès esthétique acquiert un sens, où l'intrusion du roman anglais, entre autres faits marquants, permet de penser qu'il existe quelque chose comme une évolution positive dans l'histoire des arts. En utilisant les ressources qu'offre le traducteur (du grec et du latin autant que de l'anglais) il est donc possible d'accéder à un nouvel état de plaisir pour le spectateur. Non point en calquant le théâtre français essoufflé sur un théâtre anglais archaïque et trop excessif ou «naturel», mais en allant vers un nouveau naturel, plus universel, en se servant des nouvelles connaissances et de quelques-unes des notions anglaises pour réformer le genre français en déclin, et inversement.

D'où l'intérêt de la «traduction» qui permet de prendre un certain nombre de choses tout en laissant ce qui ne correspond pas à la réforme souhaitée, en renvoyant l'archaïque et le barbare et en gardant la variété et l'accès plus libre à la vérité des caractères par exemple. Il n'est évidemment pas possible de voir d'un bon œil la dureté, la barbarie, l'indignité des grands de ce monde représentées sur scène, d'assister aux circonstances révoltantes, inutiles, aux scènes choquantes, aux discours licencieux, à la bassesse des interlocuteurs et des discours, mais le traducteur est là pour faire le tri et présenter au lecteur français une tragédie convenable, moyenne, et cependant encore surprenante. Peut-être qu'ainsi l'ennui possible du spectateur français, fondé sur l'exemplification trop systématique de la terreur et de la pitié, ou de la froide admiration, sera relevé par l'intérêt que donne le merveilleux, du moment qu'il est traité de façon «élevée» et sans compromission avec l'esprit de la populace.

Malgré le fait que le traducteur, en ce cas, se rende nécessairement coupable «d'imprudence ou de négligence aux yeux des deux nations», il n'en demeure pas moins que présenter le théâtre anglais devient légitime. Au goût jugé noble, simple, naturel par les anglais, et dur, plat, indécent par les français, aux traits révoltants et aux scènes inutiles et complaisantes, La Place substitue donc un texte moyen, un texte à lire, donné comme second, adapté de l'original et «bien au-dessous», dans tous les cas, avoue humblement le traducteur. La traduction donc à la fois un 
méta-texte, un humble essai, un premier éclairage et une matrice propre à réformer le genre.

Le texte, il le rappelle, est tout autant une traduction, une adaptation qu'une analyse :

[J'ai] crayonné par analyse tout ce qui ne tend pas directement à l'action (comme Brumoy pour les Grecs) et à l'intérêt de ces tragédies. [Je me suis] arrêté sur toutes les situations suceptibles d'une traduction tolérable pour ceux qui ne sont pas à portée de connaître par eux-mêmes les vraies beautés de l'original. ${ }^{17}$

Il a même été jusqu'à traduire «quelques unes» des scènes incriminées par «complaisance» pour l'original. Dans une structure française (toutes les scènes sont marquées en fonction des entrées et des sorties des personnages) et en mêlant les alexandrins (pour les passages importants) et la prose (pour le reste) sans forcément respecter l'alternance shakespearienne, La Place veut donc «imiter le coup de pinceau, le ton des couleurs» de l'auteur et non le détail de ses traits : c'est ainsi, et seulement ainsi, que la passion, selon lui, peut être comprise à Paris.

Ainsi, durant les cinq actes des tragédies traduites, La Place produit un texte qui enraye les débordements du discours pour le rendre adéquat à une lecture française, au nom de l'intérêt (dans tous les sens du texte, y compris celui qui consiste à utiliser ce qui peut l'être de Shakespeare pour la scène française) qu'on doit maintenant avoir pour Shakespeare et le théâtre anglais.

Pour cela, il faut s'engager sur la respectabilité du genre, et le redécoupage en scènes françaises devient nécessaire ${ }^{18}$ : la pièce française doit être lisible comme n'importe quelle autre tragédie ou comédie, c'est la règle. Une deuxième technique consiste à suggérer que, si la pièce avait été écrite en français, l'alexandrin eût été la forme de son discours. Les scènes jugées majeures, les passages «les plus beaux» viennent donc en alexandrins classiques dans la traduction pour représenter leur importance, ou leur beauté, sans que cela corresponde au changement de style de Shakespeare, puisqu'il s'agit du jugement de La Place. Le recours ponctuel à l'alexandrin permet donc d'insérer des dystiques «sublimes» à l'intérieur des résumés ou des scènes rapportés en prose. En insistant sur le sublime tragique, La Place rend alors compte des dominantes morbides ou 
cruelles des pièces, de leur côté «sombre», sans tomber dans l'excès, propre au domaine anglais.

Grâce à l'alternance de la prose et de l'alexandrin, La Place s'octroie ainsi la fonction de juge et de théoricien pour un théâtre à venir, en réhaussant ce qu'il estime digne d'être élevé au rang du style tragique et en utilisant un style "médiocre» pour rendre compte de l'intrigue ou pour en filtrer les infractions. L'alexandrin, lorsqu'il est employé par La Place, doit être à tous égards hypersignifiant; et si des scènes entières figurent, majoritairement ou en totalité, en alexandrins, c'est, de l'aveu du traducteur, qu'elles sont ainsi traduites pour leur représentativité. Le texte de Shakespeare et celui de La Place acquièrent alors leurs lettres de noblesse, ou au moins de respectabilité et de conformité à la tragédie française, au prix d'un effort théorique du lecteur et du traducteur : la traduction doit permettre la compréhension de 1'intrigue lors même qu'on ne peut exactement dire tout ce qui se passe dans le texte anglais sans heurter le public français, et dans le même temps, elle doit proposer une solution esthétique intermédiaire et productive.

\section{Commenter, résumer, juger, traduire}

Mais l'intrigue a beau être acclimatée, le discours anglais reste, et le désordre menace. Comment traduire sans trahir les canons tragiques? C'est là que se situe le nouvel enjeu. Au nom des bienséances, La Place supprime les «frivolités», exclut la sexualité, interdit l'ébauche de voyeurisme au nom du rejet nécessaire de tout goût populaire. Dans la même veine, il est amené à commenter et à dénoncer, lors de ses résumés, ce qui ne peut convenir au public continental.

Dans le tome III, et les «Analyses ou sommaire des tragédies historiques de Shakespeare non traduites», lorsqu'il en vient à Henry $V$, La Place se cantonne à un simple résumé encadré par deux jugements de valeur, l'un sur le projet anti-français de la pièce, l'autre sur les aspects comiques ou «déplacés» de la tragédie :

Shakespeare s'est plû, dans cette pièce, à faire un tableau flatteur pour sa Nation des exploits de Henry, vainqueur des François à la bataille d'Azincourt, et des malheurs de la France. On juge bien que tout y doit être outré ; et que, pour faire sa cour à la populace anglaise, l'auteur a crû ne pouvoir mieux décorer son héros, qu'en exagérant autant la 
gloire du vainqueur que la disgrâce des vaincus (p. 492).

Voilà pour l'aspect trop anglais de la tragédie, et il ajoute, afin de prendre ses distances avec les transgressions comiques tout en reconnaissant à Shakespeare des qualités admirables :

Les compagnons du fameux Falstaff, mort depuis peu dans son exil, suivent l'armée en France; et donnent encor ici matière à plusieurs scènes comiques. On $y$ voit Catherine de France essayant d'apprendre l'Anglois, d'une de ses femmes. Cette scène est en François de ce tems, et contient des choses plus que gaillardes, ainsi que celle de sa première entrevue avec Henry, son futur époux. Le monologue de Henry, au quatrième acte, dont l'armée est réduite aux dernières extrêmités de la misère, et que les François vont attaquer, contient une leçon admirable pour les rois. C'est dommage qu'elle ne soit pas mieux placée (p. 494).

De même, à propos du Roi Lear qu'il résume, La Place reprend son double jugement: reconnaître le génie de Shakespeare et simultanément, marquer les outrances et les extravagances dues au goût du siècle et du public anglais. Ainsi, Le Roi Lear, qui n'est pas digne d'être retenu dans les traductions véritables, est néanmoins noté comme une tragédie majeure de Shakespeare :

Cette pièce est peut être à la fois le chefd'œuvre du génie et de l'extravagance. C'est un contraste perpétuel, de grandeur et de bassesse, de pathétique et de frivolités, de sublime et de ridicule. Le caractère du Roi Lear, à n'en juger que par ses actions telles qu'on vient de les rapporter, doit paroître peu intéressant : un vieillard entêté, injuste envers sa fille, ingrat envers le plus zélé de ses sujets, victime d'une prédilection stupide pour deux enfants indignes de sa tendresse, et rassemblant tous les défauts de l'enfance avec ceux de la vieillesse la plus décrépite, semble devoir être un objet plus digne de mépris que 
de pitié fondée. Cependant, ce même personnage n'arrive presque jamais sur la scène, sans arracher des larmes au spectateur le moins sensible. L'excès de son malheur, l'amertume de son repentir, ses transports partant du sein de la nature, tout enfin jusqu'à la démence, remuë, intéresse, attendrit. [...]

Mais si cette vive peinture des vertus et des vices de l'humanité a attiré à Shakespeare les éloges de son siècle, la juste délicatesse du nôtre ne peut lui pardonner les indécences dont ces mêmes tableaux se trouvent souvent surchargés, ni la fidélité servile avec laquelle il s'attache à peindre également ce qui peut plaire et ce qui doit révolter. Le goût n'est pas de tous les siècles: plaignons Shakespeare de n'avoir pas vécu dans le nôtre ! (p. 518-9).

Ce type de démonstration est exactement semblable à ce qu'il était possible, depuis le XVIIe siècle (et dans les traductions de Brumoy sur le Théâtre des Grecs), de lire sur les auteurs anciens. Homère avait du génie, certes, mais il ne possédait pas le goût moderne ; Eschyle, Euripide et même parfois Sophocle, s'exprimant à la manière des Grecs, n'avaient pu bénéficier des avancées de l'art et de l'excellence des canons de la modernité.

Puisque l'empire des bienséances interdit tout excès, La Place recourt donc à plusieurs stratégies : il affaiblit le discours, ennoblit les gestes, occulte les mots et parfois même les séquences, ne fait que suggérer les actions - comme si l'on devait connaître le texte initial pour juger de la traduction - et magnifie ce qui peut l'être par l'emploi de l'alexandrin. Parfois encore, pour éviter toute difficulté, il renvoie en note à l'anglais cité mais non traduit... Enfin, dans certains cas, il représente une scène entière par le récit non d'un messager classique mais du traducteur lui-même et, conscient de ses stratégies d'évitement, le traducteur les exprime tranquillement :

Il y a des beautés dans cette scène, avec beaucoup de licences qui m'ont empêché de la traduire. ${ }^{19}$

Il y a certainement un art infini dans cette scène : mais ceux qui l'ont lue dans l'original sentiront les raisons de bienséance qui m'ont 


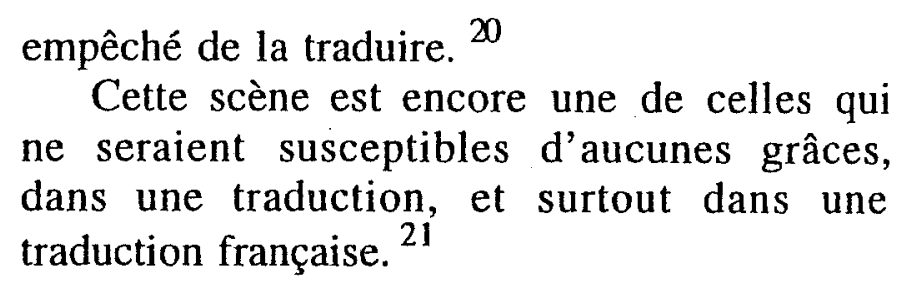

Il ne faut donc pas traduire, dit La Place, quand Shakespeare se permet d'utiliser les ressorts de la comédie pour rendre compte de la passion tragique. Au lecteur, s'il le souhaite, de prendre dans le texte-source les éléments nécessaires à son esthétique propre et à sa démonstration. Un exemple : dans sa traduction de Richard III, La Place éprouve le besoin, dans un NB liminaire, de commenter les dangers inhérents à la traduction de cette tragédie : «Cette pièce est traduite aussi littéralement qu'il est possible (du moins à l'auteur de cette traduction) de rendre en François ce que l'original a de hardi et de singulier. Ceux qui possèdent le langage de Shakespeare ne trouveront surement rien d'outré dans la manière dont on a tâché de le transmettre dans notre langue».

La Place établit donc un filtrage particulièrement net de la passion et une adaptation au vraisemblable français dans ses traductions qui lui permettent de ne pas choquer tout en informant. Le texte est donc sauvé, les personnages héroösés et la lecture de la traduction française possible. C'est un premier pas. Le second est de montrer qu'entre la tragédie anglaise et la tragédie française, un genre intermédiaire semble se dessiner, ou plutôt qu'une humble proposition théorique et pratique peut être faite pour qu'advienne un genre moyen, in between, et pour l'instant seulement propre à la lecture.

\section{La traduction, genre intermédiaire ou lieu de passage esthétique et générique ?}

Les traductions de La Place sont ainsi le résultat d'une envie et d'une peur. D'une envie de rendre lisible ce qui fait partie de la mode, tout d'abord, de rendre accessible le fonds d'une culture à un public plus large, ensuite. Une envie de réformer la tragédie, genre qui s'essouffle et qui ne pourra retrouver une quelconque vigueur qu'en élargissant son champ de références, on le voit chez Voltaire, avec Zaïre par exemple. Une nécessité enfin d'ouvrir le théâtre à des sources plus universelles, quitte à choquer les canons consacrés. Mais aussi une peur. La peur de l'hybride, une peur et une défiance envers une langue hétérogène et débridée qui laisse s'exprimer les 
sens dans leur diversité alarmante. La peur de toute une esthétique devant les transcriptions d'une passion qu'elle ne peut, à proprement parler, transcrire. La France n'est pas prête à voir l'image et le texte délirant d'une passion dévorante envahir la scène, elle ne peut, dans l'enclos des mots tragiques, observer les déchaînements du spectacle puisque la tragédie française est avant tout un discours, et finalement, doit revenir sans cesse à cet accord de vraisemblance hiérarchiquement ordonné qui caractérise encore, massivement, le théâtre français. La Place, traducteur de Shakespeare, n'a pu retranscrire qu'une ombre, n'a voulu que donner les apparences d'une sensation lointaine, et a finalement représenté le frôlement d'un texte ambigu, apeuré par la violence du texte élisabéthain, mais c'était déjà triompher d'un combat.

Mais est-ce seulement la France et ce qu'on entend, par un préjugé fort commode, par «l'esprit français», qui ne peuvent entendre «le Shakespeare» des années 1600 ? Ne sont-ce pas plutôt le théâtre et les mentalités du XVIIIe siècle ? Car dans cette Angleterre, que La Place connaît, on joue un Shakespeare modernisé, autrement dit un Shakespeare revu et corrigé au travers de Dryden, du théâtre de la Restoration, et des nouveaux drames pathétiques : un Shakespeare adapté, réécrit, que s'approprient par les auteurs, les lecteurs et les spectateurs du temps. Si bien qu'on est parfaitement en droit de penser que - même s'ils l'ont lu dans une version ancienne, ce qui n'est pas certain -, ni Voltaire, ni La Place, ni leurs héritiers, ne se sont trouvés, en Angleterre, face «au Shakespeare de 1600», mais qu'ils ont pu voir «le Shakespeare» de l'époque. Il semble ainsi que les peurs, les critiques, et le principe même de réécriture et d'adaptation, soient en partie partagés par les auteurs, les spectateurs anglais et les «traducteurs» français, et que tout cela amène à penser «Shakespeare» comme un ensemble disponible à la réforme du théâtre, d'un côté comme de l'autre de la Manche.

Ainsi, et c'est peut-être là le plus intéressant, dans le même temps qu'il traduit, s'approprie, trie et adapte le «Shakespeare» qu'il connaît, le translateur français propose un texte second, à la disposition des auteurs de son siècle et de sa nation. Il propose un texte qui tient compte de l'évolution du genre tragique et qui souhaite nourrir cette évolution de ce qu'on peut raisonnablement tirer du texte shakespearien. En cela, il ouvre un chemin pour les traducteurs et pour les dramaturges, ce dont les lecteurs de cette traduction - dont nous avons le témoignage - conviennent sans 
difficulté. Mais pour progresser, ou évoluer, l'esthétique devra, un moment, abandonner Shakespeare pour mieux y revenir ensuite.

Reprenons: en introduisant Shakespeare, La Place informe, innove et choque. Simultanément, sa traduction acquiert une autorité et devient une première référence, essentielle, à la manière de celle de Brumoy pour le théâtre des Grecs. Mais plutôt que de poursuivre la ligne de ce premier travail et de traduire ce qui ne l'a pas été - Roméo et Juliette, en particulier que l'auteur du Théâtre anglois regrette de n'avoir pas traduit -, les traducteurs du temps se tournent vers des textes anglais plus proches, chronologiquement et esthétiquement, parce qu'ils sont plus policés et moins choquants. Et c'est après ce passage par la proximité qu'on en reviendra à Shakespeare, mais autrement.

Mis à part les essais de traduction de Voltaire (le monologue d'Hamlet dans les Lettres philosophiques), et la traduction de quelques scènes de La Tempête par Destouches, en 1745, et l'ouvrage de La Place, les traductions du théâtre anglais portent, à la suite de ces textes, majoritairement sur le théâtre postérieur à Shakespeare (de la Restoration et après). Il s'agit en effet d'intéresser et d'informer le public français, autrement dit de faire le point sur ce que les anglais aiment voir jouer à la même époque, en prenant aussi pour référence Drury Lane, en triant et en cherchant à prendre ce qui conviendrait au public français («les Grands et les riches suivent assiduement les spectacles» à Paris, tandis qu'à Londres, «les personnes distinguées vont rarement à la comédie, [...] c'est donc à la bourgeoisie, même au peuple, que l'on est obligé de plaire» (Madame Riccoboni, «Avertissement», T. I, p. VIIIIX). Madame Riccoboni, dans l'avertissement de son recueil, résume bien la question :

L'unique but de cet ouvrage est d'offrir aux jeunes auteurs, qui se destinent à travailler pour le théâtre, non pas des modèles, mais un moyen d'étendre leurs idées, en mettant sous leurs yeux des scènes nouvelles et variées.

On ne traduira point de Tragédies. Le Théâtre de Monsieur de la Place, a fait connaître une partie des anciennes; et les modernes se sont extrêmement rapprochées des nôtres. La Scène Britannique ne présente plus ces horribles massacres, que la triste vérité fit supporter autrefois. Les tems 
malheureux, dont les pièces de Shakespeare retraçoient l'image, n'étoient pas encore éloignés ; on assistoit à ces tragédies avec le même sentiment qui porte à lire l'Histoire. La traduction des premiers ouvrages de ce poète, nous révolta. Les François frémirent en lisant Richard III ; tant de morts entassés dans Hamlet, nous firent penser (un peu légèrement à la vérité) que sur les bords de la Tamise, on se plaisoit à voir répandre le sang. Le tems a dissipé cette erreur, mais sans en effacer absolument la trace.

À mesure que les Anglois égayent leur scène, la nôtre se rembrunit; nous devenons sombres. Ces sensibles François, autrefois si faciles à émouvoir, dont les larmes couloient avec celles de Bérénice et Alazire, semblent dédaigner aujourd'hui des passions douces et naturelles : ils veulent moins s'intéresser, que s'attrister : on ne cherche plus à toucher leurs cours ; on s'efforce de les déchirer. Égarés par l'imagination, perdant les traces du sentiment, de la vérité, si nous ne retournons sur nos pas, il est à craindre que le goût dominant ne nous replonge dans la barbarie des premiers siècles.

Les traducteurs de l'anglais, se référant à La Place, vont donc tous chercher à représenter, dans des traductions / adaptations parfaitement acceptées, l'état de la scène anglaise. Comme La Place, ils traduisent en éliminant les excès, et comme La Place dans ses quatre derniers tomes, ils choisissent dans les productions théâtrales anglaises de la Restoration, puis du XVIIIe siècle ${ }^{22}$. Comme si La Place avait tout traduit, tout donné alors au public français, de ce qui pouvait l'être du théâtre de Shakespeare. Mais il reste l'actualité. Ainsi paraissent successivement une traduction de L'Avare de Shadwell, par Du Bocage, en 1752 ; Le Nouveau Théâtre anglois, traductions de pièces modernes, dont Lillo et Gray ( $L e$ Banquet des gueux), en 1767 ; et surtout Le Nouveau Théâtre anglois, de Madame Riccoboni, en 1769, portant sur cinq comédies jouées à Drury Lane dans les années 1755-1768. Cependant, Shakespeare n'est pas éliminé, il est devenu une sorte de référence historique, une donnée qu'il faut connaître et dont on débat, mais ses extravagances sont encore trop notables pour être véritablement reprises. 
Il faudra Ducis pour imposer à la Comédie-Française une adaptation d'Hamlet, le 30 septembre 1769 , et surtout, la grande traduction de Le Tourneur à partir de 1776 (relayée par une apologie de Shakespeare et une réponse à Voltaire de Madame de Montaigu, en 1777), pour que l'auteur anglais d'Hamlet, principalement, devienne une pomme de discorde et, de plus en plus nettement à la fin du siècle, un auteur dont le génie puisse être utile et enfin imitable.

\section{Un Hamlet intermédiaire, ou Hamlet français}

Il y a donc bien eu, à travers les traductions-adaptations de Shakespeare et du théâtre anglais du XVIIIe siècle, la production d'un genre intermédiaire, alternatif à la tragédie : un texte à lire d'abord, puis un drame tragique à l'anglaise, écrit par des Français et exploitable comme tel, utilisable aux fins de réformer la tragédie en déclin, ou trop outrageusement dominée par Voltaire. Un genre dramatique d'abord à lire, puis à jouer. À la Zaire de 1733, et après tout ce mouvement qui est au centre du XVIIIle siècle, correspond donc le Hamlet de Ducis, en 1769. C'est là un nouveau genre qui se fonde, une proposition synthétique qui réfléchit à la possiblité de produire une intrigue anglaise, mais vraisemblable, normalisée, où le pathétique domine, soutenu par la tragédie politique à la française. En cela, l'épigraphe de Ducis, tirée de La Nouvelle Hélö̈se de Rousseau, est éclairante : "Accablée d'une si cruelle perte, mon âme n'eut plus de force que pour la sentir; la voix de la nature gémissante étouffa les murmures de l'amour». Hamlet devient donc, à partir du texte de La Place - et non de celui de Shakespeare -, revu et corrigé par un lecteur de Voltaire, de Corneille, de Racine et de Rousseau, une pièce où la nature, la politique et le sentiment se partagent les ressorts de l'intrigue. Ducis, sans difficultés ni gêne, débute son avertissment par un superbe «Je n'entends point l'Anglais, et j'ai osé faire paraître Hamlet sur la scène française. Tout le monde connoît le mérite du Théâtre Anglois de M. de La Place. C'est d'après cet ouvrage précieux à la littérature que j'ai entrepris de rendre une des plus singulières tragédies de Shakespeare». Ducis, en cinq actes et en vers, compose alors un Hamlet, comme on réécrit un Edipe ou une Clytemnestre à la même époque, en interprétant la fable via la sensibilité et la codification tragique de son temps. Simultanément, il s'appuie sur les mots travestis de Shakespeare imprimés par La Place pour proposer une nouvelle forme tragique, intermédiaire entre 
la tragédie et la tragédie larmoyante : un drame sensible, politique et tragique. Hamlet devient ainsi l'histoire d'une vengeance triste, émue et nécessaire, dans une famille royale traversée par les passions. Point de comédiens, point de témoins ni de fossoyeurs, point de Rosencrantz ni de Guildenstern, point de monologue célèbre même, mais une suite d'emprunts, de citations et de collages au service de la politique telle qu'on la pense alors sur la scène de la Comédie-Française, et de l'émotion telle qu'on la lit dans le roman de Rousseau.

Dans l'enclos du «Palais des rois du Dannemarck», Hamlet doit venger son père bien qu'il aime Ophélie. Mais, chez Ducis, Ophélie est la fille de Claudius «Premier Prince du Sang» et amant de Gertrude, la mère d'Hamlet - et non la fille de Polonius qui n'est ici qu'un «Prince Danois» au service de Claudius. Dès lors, la vieille question du Cid revient sur le devant de la scène :

Oph. Vous, massacrer mon père ?

Ham.

Il m'a privé du mien.

Hamlet est alors le Hamlet de La Place, plus Le Cid, plus L'Orestie et Andromaque, plus surtout la Clytemnestre de Lauraguais qu'on a jouée en 1761 dans le même théâtre... mais avec larmes, sentiment, cœur et émotion: Hamlet pleure sur le sort d'Ophélie et sur le sien propre, pris entre le devoir et l'amour. Lorsque sa mère s'évanouit lorsqu'il l'accuse d'avoir empoisonné son père et qu'elle ne peut jurer de son innocence devant l'urne du défunt, Hamlet vacille. Lorsqu'Ophélie doit jurer allégeance à son père alors qu'elle aime encore Hamlet, elle s'émeut. Cependant, nous sommes en France, là où la tragédie politique excelle. Ophélie, in extremis, se défend contre son père en invoquant le fait qu'on ne peut tuer un roi, fût-il dangereux, fût-il un futur assassin : c'est au nom de l'interdiction du régicide que la douce Ophélie résiste à Claudius et qu'elle préserve Hamlet, ce jeune roi pris dans les rets de sa mère-régente; c'est encore au nom de cette interdiction majeure qu'elle s'offre en sacrifice. On n'attente pas sans dommage à la vie des rois : Damiens, en 1757, en avait fait la terrible et pédagogique expérience. Ainsi, lorsque Claudius forme une conjuration pour tuer Hamlet, roi de Danemark, et prendre le pouvoir en imposteur tyrannique, Hamlet vient seul, découvre horrifié le cadavre de sa mère poignardée par son amant, mais agit, 
et poignarde à son tour Claudius. Les conjurés, qui fondent sur le roi, s'arrêtent : il ne faut pas «lever sur les rois un poignard homicide».

Tout pourrait bien finir, comme chez Corneille, d'autant qu'Hamlet pleure sur son triste destin, mais tout s'arrête, comme si Ducis n'avait pas osé l'impossible transformation de la pièce en tragi-comédie à fin heureuse, ou en drame. Ophélie entre, vivante, pour clore la tragédie, et Hamlet lui déclare :

Ham Je t'adore et te perds. Ce poignard seul me reste Mais je suis homme et roi. Réservé pour souffrir, Je saurai vivre encor; je fais plus que mourir.

[fin de la pièce]

Suspendue, la «tragédie» sauve Ophélie et transfigure le prince du Danemark en roi de sacrifice, éternellement ému, souffrant, aimant, infiniment larmoyant, mais régnant. Incroyable rhapsodie à nos yeux, exactement comme les traductions de l'époque : la tragédie de Shakespeare, via le texte de La Place, la longue chaîne des «Théâtres anglois» et la tragédie française du temps, enfin réécrite par Ducis, propose donc une synthèse, doublée d'une recherche générique. On dira qu'elle est faible, ou «froide». Sans conteste, et l'histoire du théâtre ne lui a pas réservé le succès qu'elle attendait. Mais c'était un essai, un moment nécessaire pour que Shakespeare, enfin, paraisse au grand jour. On sait qu'il faudra encore attendre, tâtonner, essuyer bien des réécritures et des scandales - dont celui de 1829, avec Le More de Venise de Vigny -, tant le public français, nourri de vraisemblable et gavé de hiérarchie générique, a du mal a admettre le texte shakespearien. Les traductions témoignent, évoluent, choisissent, et simutanément agissent sur la littérature parce qu'elles trahissent, nécessairement. Elles savent bien que l'écart des langues et des littératures est heureusement irrémédiable.

Christian B IE T 


\section{NOT E S}

${ }^{1}$ Pierre-Antoine de La Place, Le Théâtre anglois, Londres, 1746-1749.

Tome I. Discours sur le théâtre anglois (p. I à CXVIII), Vie de Shakespeare, à partir des indications de Rowe et de Pope que cite La Place dans son texte (p. CXIX à CXLIII), Othello ou le More de Venise, Henry VI, roi d'Angleterre.

Tome II. La Vie et la mort de Richard III, Hamlet, prince de Danemark, Macbeth.

Tome III. Shakespeare : Cymbeline, Jules César, Antoine et Cléopâtre, Analyses ou sommaire des tragédies historiques de Shakespeare non traduites.

Tome IV. Shakespeare : Timon ou le Misanthrope, Les Femmes de bonne humeur ou les Commères de Windsor; Fletcher : La Pucelle ; Analyses ou sommaire des tragi-comédies ou des comédies de Shakespeare non traduites.

Tome V. Johnson (Ben) : Catilina ; Rowe : La Belle pénitente ; Otway : Venise sauvée (nombreuses traductions durant le XVIIIe siècle, jouée à la Comédie-Française en 1746).

Tome VI. Dryden: Aureng-Zeb ou le Grand Mogol; Congreve : L'Épouse en deuil ; Rowe : Tamerlan.

Tome VII. Hughes : Le Siège de Damas; Young : Busiris, roi d'Égypte ; Congreve : Amour pour amour.

Tome VIII : Southerne : L'adultère innocent; Addison : Caton; Steele : Les Funérailles ou le deuil à la mode.

${ }^{2}$ P. IX, «Discours sur le théâtre anglais» de 1746, in Le Théâtre anglois.

${ }^{3}$ Voir Ch. Biet, «La peste noire et le traducteur : la traduction d'Othello en France au début du XVIIIe siècle», in La Jalousie (Colloque de Cerisy), sous la dir. de Frédéric Monneyron, L'Harmattan, 1996, et Christian Biet, «Traduire Sénèque et Shakespeare au XVIIIe siècle», in Texte(s) et intertexte(s), études réunies par E. Le Calvez et M.-C. Canova-Green, Rodopi, Amsterdam-Atlanta, 1997.

${ }^{4}$ Voltaire, Discours de $M$. de Voltaire à sa réception à l'Académie Française, 9 mai 1746.

${ }^{5}$ Voltaire, Lettres philosophiques, «sur la tragédie», 1734.

${ }^{6} \mathrm{P}$. III du «Discours sur le théâtre anglais» de 1746, in Le Théâtre anglois, op. cit.

7 P. II, ibid.

${ }^{8}$ Voir le premier chapitre de Ch. Biet, Edipe en monarchie, Klincksieck, 1994.

${ }^{9}$ P. II du «Discours», op. cit. 
${ }^{10}$ P. IX, ibid.

11 P. IX, ibid.

12 P. XXI, ibid.

${ }^{13}$ P. XXXIII, ibid.

${ }^{14}$ Selon Riccoboni : Réflexions historiques et critiques sur les différents théâtres de l'Europe, 1718, cité par La Place.

${ }^{15}$ Inversement, Phèdre, Mithridate, Andromaque, l'Avare et le Misanthrope doivent être surchargés d'intrigues secondaires et de spectacle s'ils veulent être représentés à Londres.

${ }^{16} \mathrm{P} . \mathrm{XXI}$, ibid.

17 P. CXI, ibid.

${ }^{18}$ Brumoy et les traducteurs l'avaient déjà noté, rien d'original donc ici.

19 P. 109, lère sc. de l'acte IV, Othello-Iago.

${ }^{20}$ P. 112. Acte IV, sc. 5, Iago-Cassio.

${ }^{21}$ P. 136, Acte IV, sc. 20, Desdémone, Émilie.

2 - Du Bocage, traduit L'Avare de Shadwell, en 1752.

- Le Nouveau Théâtre anglois (Anonyme) dont Gray (Le Banquet des gueux), 1767.

- Madame Riccoboni, Le Nouveau Théâtre anglois, 1769. Cinq comédies jouées à Drury Lane dans les années 1755-1768: Tome I. The Foundling, L'Enfant trouvé, comédie en cinq actes par Edward Moore (Drury-Lane, 1755) ; The way to keep him, La Façon de le fixer, comédie en cinq actes par M. Murphy (Drury-Lane, 1761) ; Tome II. False delicacy, La Fausse délicatesse, comédie en cinq actes par M. Hugh Kelly (Drury-Lane 1768) ; The jealous wife, La femme jalouse, comédie en cinq actes par George Colman, écuyer (d'après Tom Jones, Drury-Lane, 1763) ; The deuce is in him, Il est possédé, comédie anonyme en deux actes, d'après un conte de Marmontel (Drury-Lane, 1764).

- La Vasse (Baronne de), 1784, 6 volumes de traduction de pièces de la fin du XVIIe siècle et surtout du XVIIIe, mais pas de Shakespeare. 